\title{
Application of Extension Theory in the Assessment of Equipment Procurement Risk
}

\author{
Xianpei Jiang ${ }^{1, a^{*}}$, Li Pu ${ }^{1, b}$, Caoshan Wang ${ }^{1, c}$ and Dehua Ou Yang ${ }^{1, d}$ \\ ${ }^{1}$ College of Equipment Engineering, Engineering University of CAPF, Xi'an710086, China \\ a18192335913@163.com, b314576171@qq.com, 515026339@qq.com, doydh2013@126.com
}

Keywords: Equipment acquisition; Risk assessment; Extension theory; Anti-riot armored vehicle

\begin{abstract}
Depending on the cause of equipment acquisition risk, a multi-level evaluation model is to be built based on the extension theory. The simple dependent function and the similar coefficient methods are used to determine the level of the index weight objectively and scientifically, and make extension assessment for the equipment acquisition risk assessment system. In the end, the feasibility, objectivity and validity of this system is proved by an example, which is applied to a certain type of anti-riot armored vehicle of CAPF.
\end{abstract}

\section{Introduction}

The weapon and equipment procurement is a basic method of equipment acquisition, which plays a precedent role in the equipment whole life cycle, and also covering the whole process from development to use of the equipment. The whole process is of large scale, wide range and involving multiple departments. And also each step has complicated uncertain factors. The characteristics of complexity and one-time feature put forward higher requirements on equipment procurement, thus the risks of procurement work has become increasingly serious. Based on the current situation, in order to realize low consumption and high return of acquisition work, it is necessary to implement effective assessment of procurement risk, of which the selection of scientific assessment methods is particularly important.

The assessment of equipment procurement risk has developed for many years in China and has obtained a certain effect. For example, Hua Zhang [1] and others study the equipment procurement risk by combining BP algorithm; Xusheng Gan [2] and others do the researches by using FAHP (Fuzzy Analytic Hierarchy Process); Wenxin Li [3] and others evaluate the equipment procurement risk by using the method of improving risk matrix. However, all the methods mentioned above require large data, and are easily influenced by subjective experience, lack of flexibility and have difficulty to solve qualitative problems. The extension theory proposed by Professor Wen Cai takes matter-element as the basic unit of description, using qualitative and quantitative methods to solve complex problems, and taking the object and the characteristics of object and its corresponding value as a whole to study [4].Using correlation function to expand the qualitative problem of "belonging to or not belonging to" to a quantitative description [5]. Its subject characteristics completely accord with the behavioral characteristics of equipment procurement. Based on the extension theory and combining simple correlation function and similar coefficient method, the author analyzes the specific applications in equipment procurement risk assessment of extension theory.

\section{Equipment Procurement Risk Information Matter-element System}

Equipment procurement is of wide range and can be influenced by multiple in factors, including subjective factors, objective factors, internal factors and external factors, and many other aspects. Based on the matter-element extension theory and combining the actual situation in the Army, this paper takes the equipment procurement risk as a primitive; according to the cause of the risk[6],to expand these two object primitives of external factors and internal factors, and then to respectively 
expand the upper two primitives. By using this analogy, it can constitute a top-down matter-element extension. As shown in Fig. 1.

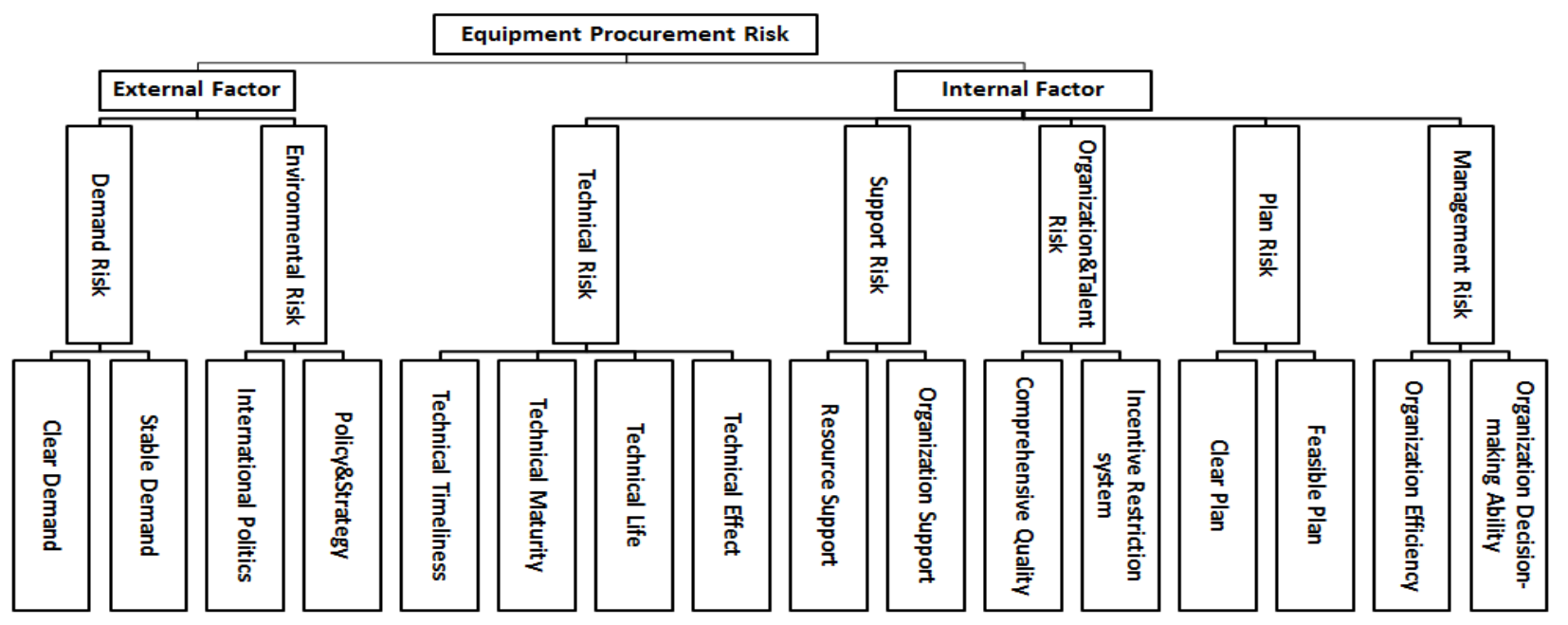

Figure 1. Extension model of equipment procurement risk assessment

\section{The Fuzzy Matter-element Model of Equipment Procurement Risk}

Equipment procurement risk assessment can be achieved through the matter-element exchange among the elements in the extension domain, with the matter-element extension of procurement risk assessment as the basis and extension domain of risk assessment as the research subject. The modeling process is as follows:

The Matter-element of Equipment Procurement Risk Assessment. According to the matter-element theory, taking the orderly three-element composite $\mathrm{R}=(\mathrm{N}, \mathrm{C}, \mathrm{V})$ as the basic element of object research, which are the object name, characteristics, and value. $\mathrm{N}$ represents the object name (This paper refers to the fuzzy information matter-element of equipment procurement risk assessment). $\mathrm{C}$ represents the name of characteristics (This paper refers to the name of indexes). $\mathrm{V}$ represents the value of $\mathrm{N}$ regarding $\mathrm{C}$ (This paper refers to the corresponding index value).

The Matter-element Model of Procurement Risk. Assessment Grading Standard. After looking up to the document references and combining the commonly used grading standards, according to the value and dereferencing principle (which is when the value is smaller, the risk will be less), there divides 5 grades $N_{p}$, which are lower risk $N_{0}$, low risk $N_{1}$, middle risk $N_{2}$, high risk $N_{3}$, higher risk $N_{4}$.

Confirming Classical Domain [7]. Set $N_{j}(j=1,2, \cdots, \mathrm{m})$ as the grades divided for the index of equipment procurement risk assessment. The total quantity of the grades is $j . C_{i} \quad(i=1,2, \cdots, n)$ is the No.1 index. $C_{i k}\left(k=1,2, \cdots, n_{i}\right)$ represents the lower assessment index of $C_{i}$. So we have this formula:

$$
R_{j}=\left[\begin{array}{ccccc}
N & N_{1} & N_{2} & \cdots & N_{m} \\
C_{i k} & V_{i 1} & V_{i 2} & \cdots & V_{i m}
\end{array}\right]=\left[\begin{array}{cccc}
N & N_{1} & \cdots & N_{m} \\
C_{11} & \left\langle a_{11}, b_{11}\right\rangle & \cdots & \left\langle a_{1 m}, b_{1 m}\right\rangle \\
\vdots & \vdots & & \vdots \\
C_{i k} & \left\langle a_{i 1}, b_{i 1}\right\rangle & \cdots & \left\langle a_{i m}, b_{i m}\right\rangle \\
\vdots & \vdots & \vdots & \vdots \\
C_{n n_{i}} & \left\langle a_{n 1}, b_{n 1}\right\rangle & \cdots & \left\langle a_{n m}, b_{n m}\right\rangle
\end{array}\right]
$$


In this formula, $V_{i j}=\left\langle a_{i j}, b_{i j}\right\rangle$ represents the value range of $N_{i}$ regarding $C_{i k}$, which refers to corresponding numerical range of each index on each grade.

Confirming Joint Domain. Joint domain refers to value range of each index of procurement risk in all grading levels.

$$
R_{p}=\left(P, C_{i k}, V_{i p}\right)=\left[\begin{array}{cccc}
P & C_{11} & \cdots & \left\langle a_{1 p}, b_{1 p}\right\rangle \\
& \vdots & & \vdots \\
& C_{i k} & \cdots & \left\langle a_{2 p}, b_{2 p}\right\rangle \\
\vdots & & \vdots \\
& C_{n n_{i}} & \cdots & \left\langle a_{n p}, b_{n p}\right\rangle
\end{array}\right]
$$

In this formula, $\mathrm{P}$ refers to all the grades in this index system. $V_{i p}=\left\langle a_{i j}, b_{i j}\right\rangle$ refers to the numerical range of $P$ regarding $C_{i}$, which is the joint domain of $P$.

The Confirmation of Matter-element under Test. The matter-element under test refers to that through assessing the procurement risk to get each index value $S$, and then use matter-element form to show the index value:

$$
R_{S}=\left[\begin{array}{ccc}
S & C_{11} & v_{11} \\
& \vdots & \vdots \\
& C_{i k} & v_{i k} \\
\vdots & \vdots \\
& C_{n n_{i}} & v_{n n_{i}}
\end{array}\right]
$$

Single Index Correlation Degree. Single index correlation degree refers to the value range degree of a certain index's assessed value. The mathematical meaning is the distance between the dot on the axis and its value range. Here to set the correlation function as (The meaning of each symbol is same as what mentioned above).

$$
K_{j}\left(v_{i}\right)=\left\{\begin{array}{c}
\frac{\rho\left(v_{i}, V_{i j}\right)}{\rho\left(v_{i}, V_{i p}\right)-\rho\left(v_{i}, V_{i j}\right)}\left(V_{i} \not \subset\left[a_{i j}, b_{i j}\right]\right) \\
-\rho\left(v_{i}, V_{i j}\right)\left(V_{i} \in\left[a_{i j}, b_{i j}\right]\right)
\end{array}\right.
$$

Here refers to the distance between Dot and Section. The calculation formula is:

$$
\rho(x,\langle a, b\rangle)=\left|x-\frac{a+b}{2}\right|-\frac{b-a}{2}
$$

Multi-level Extension Assessment. When conducting the Level 1 index assessment, get the correlation degree of Level 1 index in each risk degree.

$$
K_{j}(v)=\sum_{i=1}^{n} \omega_{i} K_{j}\left(v_{i}\right)
$$

When conducting the Level 2 index assessment, get the correlation of different risk degree of the object under test.

$$
K_{j}(S)=\sum_{i=1}^{n} \omega_{i k} K_{j}(v)
$$


Comprehensive Assessment. The bigger the correlation degree of Level $j$ means the procurement risk is more in line with this level. And that which has the biggest conformity degree is the risk level of this procurement. If $K_{j 0}(S)=\max _{j \in(1,2, \cdots, k)} K_{j}(S)$, then the level of $S$ is $j$.

The eigenvalues of grade variables formula of $S$ is:

$$
j^{*}=\frac{\sum_{j=1}^{m} j \cdot \overline{K_{j}}(S)}{\sum_{j=1}^{m} \overline{K_{j}}(S)}
$$

In this formula, $\overline{K_{j}}(S)=\frac{K_{j}(S)-\min _{j} K_{j}(S)}{\max _{j} K_{j}(S)-\min _{j} K_{j}(S)}$. This leveled characteristic value of $j^{*}$ is the extent of the object under test $S$ tending to another level. For example, $j^{0}=1.3, j^{*}=2.3$ means the object under test belongs to Level 1, but tending to Level 2.

Overall Assessment Model. Based on the risk assessment of different periods, we can make the dynamic tracing evaluation of the risk system. So, when the value and degree of $j^{*}$ is very high, and has the tendency to increase, it means the risk is big and will become bigger; when the degree of $j^{*}$ is low, the variation is small and the trend is smooth, it means in a certain period the risk will be low.

\section{The Confirmation of Assessment Index Weighting}

There are many kinds of methods to confirm the index weighting, mainly are Expert scoring method, Statistical analysis method, Principle component analysis and so on. In order to make the weighting value to meet the requirement of objectivity and dynamic, this paper utilizes simple correlation function to confirm weighting[8].Here to set the weighting of the equipment procurement risk assessment index $C_{i}$ as $\omega_{i}$, and $\sum_{i=1}^{n} \omega_{i}=1 . \omega_{i k}$ is the weighting of its lower subsidiary index $C_{i k}$, and $\sum_{i=1}^{n} \omega_{i k}=1$

Weighting Confirmation of Level 1 Index.

$$
r_{i j}\left(v_{i}, V_{i j}\right)=\left\{\begin{array}{l}
\frac{2\left(v_{i}-a_{i j}\right)}{b_{i j}-a_{i j}}, v_{i} \leq \frac{a_{i j}+b_{i j}}{2} \\
\frac{2\left(b_{i j}-v_{i}\right)}{b_{i j}-a_{i j}}, v_{i} \geq \frac{a_{i j}+b_{i j}}{2}
\end{array}(i=1,2 \cdots n, j=1,2 \cdots m)\right.
$$

And $v_{i} \in V_{i p}(i=1,2 \cdots n)$, then :

$$
r_{i j_{\max }}\left(v_{i}, V_{i j_{\max }}\right)=\operatorname{Max}_{j}\left\{r_{i j}\left(v_{i}, V_{i j}\right)\right\}
$$

If the level that the index falls is higher, the index should be given more weighting. So:

$$
r_{i}= \begin{cases}j_{\max } \times\left(1+r_{i j_{\max }}\left(v_{i}, V_{i j}\right)\right) & , \text { 当 } r_{i j_{\max }}\left(v_{i}, V_{i j}\right) \geq-0.5 \\ j_{\max } \times 0.5 & , \text { 当 } r_{i j_{\max }}\left(v_{i}, V_{i j}\right)<-0.5\end{cases}
$$

If the level that the index falls is lower, the index should be given less weighting. So: 


$$
r_{i}= \begin{cases}\left(m-j_{\max }+1\right) \times\left(1+r_{i j_{\max }}\left(v_{i}, V_{i j}\right)\right) & , \text { 当 } r_{i j_{\max }}\left(v_{i}, V_{i j}\right) \geq-0.5 \\ \left(m-j_{\max }+1\right) \times 0.5 & \text {, 当 } r_{i j_{\max }}\left(v_{i}, V_{i j}\right)<-0.5\end{cases}
$$

Then the weighting of index $C_{i}$ will be:

$$
\omega_{i}=r_{i} / \sum_{i=1}^{n} r_{i}
$$

Weighting Confirmation of Level 2 Index. According to Eq.9, we can get the optimal evaluation matrix $S_{k j}^{i}$ of each index in Level 2.

$$
S_{k j}^{i}=\left[\begin{array}{ccc}
S_{11}^{i} & \cdots & S_{1 j}^{i} \\
\vdots & & \vdots \\
S_{n_{i} 1}^{i} & \cdots & S_{n_{i} j}^{i}
\end{array}\right]
$$

If $a_{0 k}=1 / n$, then we can get the equal weight vector $a_{0}=\left[\begin{array}{llll}\frac{1}{n_{i}} & \frac{1}{n_{i}} & \cdots & \frac{1}{n_{i}}\end{array}\right]$ under the equal weight condition. So the Level 2 indexes' comprehensive optimal evaluation vector $S_{i j}$ is:

$$
S_{i j}=a_{0 k} S_{k j}^{i}
$$

$S_{i j}$ is believed that it can reflect the general assessment level of the to be assessed object about Index $C_{i}$. The similarity between $S_{k j}^{i}$ (single-stage optimal evaluation vector )and $S_{i j}$ (comprehensive optimal evaluation vector) reflects the reflection ability of index $C_{i k}$ to the general condition of $C_{i}$. The closer of $S_{k j}^{i}$ and $S_{i j}$, it means the stronger reflection ability of $C_{i k}$ to $C_{i}$, and the bigger weight of $C_{i k}$.

The closeness of the 2 non-negative vectors can be compared by using similar coefficient method. Here, we can set the similar coefficient as $r_{i k}$, then:

$r_{i k}=\frac{1}{n_{i}} \sum_{j=1}^{m} S_{k j}^{i} S_{i j}^{\mathrm{T}}$

Then $\omega_{i k}$ (weighting of Level 2 index) will be:

$\omega_{i k}=r_{i k} / \sum_{k=1}^{n_{i}} r_{i k}$

\section{Case Study}

This paper takes the risk assessment of the purchasing of a certain type riot vehicle by Armed Police Force as the example to check the feasibility and applicability of assessing based on the extension theory.

According to the extension theory and procurement risk assessment index system, to get the classical and joint domain, and the present value under test, like what shows in Table 2. The data source comes from the current mission requirements of the Armed Police Force, and the comparison of domestic and overseas riot vehicle specification of similar type, then to get the present value of each assessment index. See the last line in Table 1. 
Table 1 Risk assessment index system and index value of the purchasing of a certain type riot vehicle

\begin{tabular}{|c|c|c|c|c|c|c|c|}
\hline $\begin{array}{c}\text { Index } \\
\text { Level } 1\end{array}$ & $\begin{array}{c}\text { Index } \\
\text { Level } 2\end{array}$ & Lower Risk & Low Risk & Middle Risk & High Risk & Higher Risk & $\begin{array}{c}\text { Present Value } \\
\text { Under Test }\end{array}$ \\
\hline \multirow[t]{2}{*}{ Demand Risk } & Clear Demand & $\langle 0.10,0.30\rangle$ & $\langle 0.30,0.50\rangle$ & $\langle 0.50,0.70\rangle$ & $\langle 0.70,0.85\rangle$ & $\langle 0.85,1\rangle$ & 0.20 \\
\hline & Stable Demand & $\langle 0.10,0.30\rangle$ & $\langle 0.30,0.50\rangle$ & $\langle 0.50,0.70\rangle$ & $<0.70,0.85>$ & $<0.85,1\rangle$ & 0.15 \\
\hline \multirow[t]{2}{*}{$\begin{array}{c}\text { Environmental } \\
\text { Risk }\end{array}$} & $\begin{array}{c}\text { International } \\
\text { Politics }\end{array}$ & $<0,0.20\rangle$ & $<0.20,0.40>$ & $\langle 0.40,0.60\rangle$ & $<0.60,0.80>$ & $<0.80,1>$ & 0.32 \\
\hline & Policy\&Strategy & $<0.10,0.25>$ & $<0.25,0.45>$ & $<0.45,0.60\rangle$ & $<0.60,0.80>$ & $<0.80,1>$ & 0.22 \\
\hline \multirow[t]{4}{*}{ Technical Risk } & $\begin{array}{l}\text { Technical } \\
\text { Timeliness }\end{array}$ & $\langle 0,0.15\rangle$ & $<0.15,0.35>$ & $<0.35,0.60>$ & $<0.60,0.80>$ & $<0.80,1>$ & 0.63 \\
\hline & Technical Maturity & $<0,0.15>$ & $\langle 0.15,0.35>$ & $<0.35,0.60\rangle$ & $\langle 0.60,0.80\rangle$ & $\langle 0.80,1\rangle$ & 0.56 \\
\hline & Technical Life & $\langle 0,0.15\rangle$ & $\langle 0.15,0.35>$ & $\langle 0.35,0.60\rangle$ & $<0.60,0.80\rangle$ & $<0.80,1>$ & 0.70 \\
\hline & Technical Effect & $<0,0.15\rangle$ & $\langle 0.15,0.35\rangle$ & $\langle 0.35,0.60\rangle$ & $\langle 0.60,0.80\rangle$ & $<0.80,1>$ & 0.37 \\
\hline \multirow[t]{2}{*}{ Support Risk } & Resource Support & $\langle 0,0.20\rangle$ & $\langle 0.20,0.40\rangle$ & $<0.40,0.60\rangle$ & $<0.60,0.80\rangle$ & $<0.80,1>$ & 0.53 \\
\hline & $\begin{array}{l}\text { Organization } \\
\text { Support }\end{array}$ & $<0,0.20\rangle$ & $<0.20,0.40>$ & $<0.40,0.60>$ & $<0.60,0.80>$ & $<0.80,1>$ & 0.45 \\
\hline \multirow[t]{2}{*}{$\begin{array}{l}\text { Organization\& } \\
\text { alent Risk }\end{array}$} & $\begin{array}{c}\text { Comprehensive } \\
\text { Quality }\end{array}$ & $\langle 0,0.20\rangle$ & $<0.20,0.40>$ & $<0.40,0.60>$ & $<0.60,0.80>$ & $<0.80,1>$ & 0.37 \\
\hline & $\begin{array}{c}\text { Incentive } \\
\text { Restriction system }\end{array}$ & $\langle 0,0.20\rangle$ & $\langle 0.20,0.40\rangle$ & $<0.40,0.60>$ & $<0.60,0.80>$ & $<0.80,1>$ & 0.52 \\
\hline Plan Risk & $\begin{array}{c}\text { Clear Plan } \\
\text { Feasible Plan }\end{array}$ & $\begin{array}{l}<0,0.15> \\
\langle 0,0.15>\end{array}$ & $\begin{array}{l}<0.15,0.35> \\
\langle 0.15,0.35>\end{array}$ & $\begin{array}{l}<0.35,0.60> \\
<0.35,0.60>\end{array}$ & $\begin{array}{l}<0.60,0.80> \\
<0.60,0.80>\end{array}$ & $\begin{array}{l}<0.80,1> \\
<0.80,1>\end{array}$ & $\begin{array}{l}0.45 \\
0.43\end{array}$ \\
\hline \multirow[t]{2}{*}{$\begin{array}{l}\text { Management } \\
\text { Risk }\end{array}$} & $\begin{array}{l}\text { Organization } \\
\text { Efficiency } \\
\text { Organization }\end{array}$ & $\langle 0,0.20\rangle$ & $<0.20,0.40>$ & $<0.40,0.60>$ & $<0.60,0.80>$ & $<0.80,1>$ & 0.54 \\
\hline & $\begin{array}{c}\text { Decision-making } \\
\text { Ability }\end{array}$ & $\langle 0,0.20\rangle$ & $<0.20,0.40>$ & $<0.40,0.60>$ & $<0.60,0.80>$ & $<0.80,1>$ & 0.35 \\
\hline
\end{tabular}

The Weighting Calculation of Each Index. First, according to Eq.9 to get the correlation function of the corresponding classical domain, to which the second level index of $C_{i}$ belong. When the correlation function falls to the bigger level, the bigger the weighting is. By using Eq. 11 and Eq. 13, we can get the weighting $\omega_{i}=[0.108,0.097,0.324,0.092,0.195,0.097,0.086]$. According to Eq. 9, we can get the optimal evaluation matrix $S_{k j}^{i}$ of each index in Level 2.

According to Eq.15, get the multi-index comprehensive evaluation matrix under equal weighting condition. $C_{1}$, the first index of $C_{i}$, has 2 elements, which means $a_{0 k}=1 / 2$. So, $S_{1 j}=\left[\begin{array}{lllll}0.75 & -1.25 & -3.25 & -7.00 & -9.00\end{array}\right]$. Then according to Eq.16, get similarity coefficient $r_{1 k}=\left[\begin{array}{ll}68.2086 & 74.4789\end{array}\right]^{\mathrm{T}}$. So by Eq.17 to get $C_{1}$ 's lower index weighting $\omega_{1 k}=\left[\begin{array}{ll}0.4781 & 0.5219\end{array}\right]^{\mathrm{T}}$.

By this analogy, get the rest weighting respectively:
$\omega_{2 k}=\left[\begin{array}{ll}0.4297 & 0.5702\end{array}\right], \omega_{3 k}=\left[\begin{array}{lll}0.2772 & 0.2475 & 0.3069\end{array}\right.$
$0.1684], \omega_{4 k}=\left[\begin{array}{ll}0.4975 & 0.5025\end{array}\right]$
$\omega_{5 k}=[0.5355$
$0.4645], \omega_{6 k}=[0.5023$
$0.4977], \omega_{7 k}=\left[\begin{array}{ll}0.4514 & 0.5486\end{array}\right]$.

Index Evaluation. According to Eq. 6, conduct Level 1 evaluation, and get $K_{j}(v)$ :

$$
K_{j}(v)=\left[\begin{array}{ccccc}
0.0739 & -0.6304 & -0.8152 & -0.8766 & -0.9014 \\
-0.1001 & -0.0797 & -0.4606 & -0.6339 & -0.7303 \\
-0.5373 & -0.3733 & -0.0842 & -0.0462 & -0.3418 \\
-0.3847 & -0.1581 & 0.0600 & -0.1901 & -0.4014 \\
-0.3544 & -0.0768 & -0.0030 & -0.2716 & -0.4590 \\
-0.3972 & -0.1694 & 0.0900 & -0.2666 & -0.4499 \\
-0.4716 & -0.2807 & -0.0415 & -0.0779 & -0.3564
\end{array}\right]
$$

On this basis and according to Eq.7, conduct Level 2 evaluation, and get comprehensive evaluation value, which is:

$$
K_{j}(S)=\left[\begin{array}{lllll}
-0.3594 & -0.2669 & -0.1499 & -0.2741 & -0.4797
\end{array}\right]
$$


Among the comprehensive evaluation value $K_{j 0}(S)=\max _{j \in(1,2, \cdots, k)} K_{j}(S)=-0.1499$. We can make the preliminary judgement that this equipment procurement risk belongs to the third risk level- Middle Risk. In order to get the accurate result, according toEq.8, we can get $j^{*}=2.715$. Judging from this, the risk is between Low Risk and Middle Risk level, tending to Middle Risk. Judging by the present value of single index, there's big risk on technical and support risk. We should pay more attention onto these two aspects.

\section{Summary}

To establish the multi-level extension model of equipment procurement risk assessment by taking the equipment procurement risk source a primitive and based on the qualitative and quantitative aspects. By using simple correlation function and similarity coefficient method, the weighting can be given to each index scientifically and objectively. Through the deep and careful study and analysis of each index in procurement assessment model, we can not only figure out the overall assessment results, but also can realize effective supervision to each sub-step. When unexpected situation appears, the problems can be found in time.

For different object and present value, the assessment system will give different weighting to the indexes, timely reflect the current risks, and realize dynamic monitoring on research targets. Meanwhile, the introduction of similarity coefficient method solves the multi-level assessment which the normal extension theory can't realize, and expands the application space of extension theory.

Data is the foundation in the assessment process. So, data processing is very important. The whole assessment is conducted by taking the classical domain, joint domain and present value under test as the core. To ensure the accuracy of assessment results, the acquirement of present value should be reliable. And the division of classical domain is also one of the key points.

\section{References}

[1] Hua Zhang, Hailin Dong, Jun Ang. Study on BP Neural Networks Model for Evaluating Equipment Acquisition Risk [J]. Fire Control \& Command Control, 2009, 34(4):100-103.

[2] Xusheng Gan, Huizi Duanmu, Hailong Gao, Meiyi Wang. Risk Assessment of Equipment Purchasing Based on Fuzzy Analytic Hierarchy Process [J]. Fire Control \& Command Control, 2011, 36(9):60-64.

[3] Wenxin Li, Xiong Pan, Fan Luo. Improved Risk Matrix and Its Application in Acquisition Risk Assessment of Certain Type Military Equipment [J]. Ordnance Industry Automation, 2011, 30(9):11-15.

[4] Wen Cai, Chunyan Yang, Bin He. Preliminary Extension Logic [M]. Beijing, Science Press, 2003.

[5] Kaixuan Cui, Quan Shi, Qiwei Hu, etc. Training Effect Evaluation of Equipment Maintenance Support Serviceman Based on Interval Number and Extension Theory [J]. Ordnance Industry Automation, 2013, 32(2):12-16.

[6] Zhongmin Li, Shuchun Tang, Jun Li, Jian Chen. Study on Acquisition Risk Identification for Weapon and Equipment Based on the Full Life Cycle [J].Engineering Science, 2005, 7(12):49-53.

[7] Wen Cai. Matter-element Model and Its Application [M]. Beijing: Scientific and Technical Documentation Press, 1994:78-94.

[8] Dongdong Qiu, Jielin Zhang. Evaluation of Urban Public Transit Hub Stations Based on Multi-level Extension Evaluation Method [J]. Journal of Wuhan University of Technology, 2012, 36(3):519-522. 